\title{
PROGRESS OF THE OPTICAL VERY LARGE ARRAY
}

\author{
A. LABEYRIE,
}

D. MOURARD, F. MORAND, L. ARNOLD, B. DEJONGHE, J. DEJONGHE, C. CAZALE, G. LEMAITRE, D. VERNET, A. BLAZIT, J. TEXEREAU, J. KESSIS, J. P. RAMBAUT

OCA URA CNRS 1361

F-06460 Saint Vallier de Thiey

\begin{abstract}
.
The construction of a prototype telescope is now reaching the stage of mirror polishing and building the active cell. Three blanks have already been produced. Two polishing methods are tested, utilizing the stressing technics, or involving excentered polishing strokes. A working model of one leg of the telescope translator has been built.
\end{abstract}

Key words: interferometry, thin mirrors, active optics, robots

\section{Introduction}

The Optical Very Large Array project is an attempt at building an imaging interferometer truly optimized for high-yield observations.

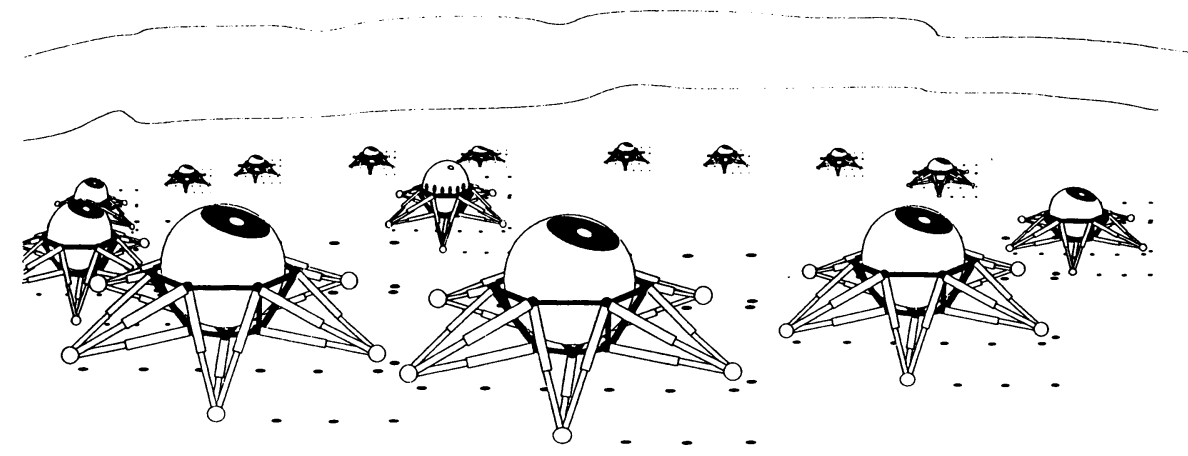

Figure 1: The Optical Very Large Array will have many telescopes, movable during the observation to maintain the temporal coherence. The light which they capture is aimed in the form of collimated coudé beams towards the central station where the images are superposed. Laser beams from the central station, aimed towards the center of each telescope, provide distance measurements needed to control the geometry with a few microns of accuracy. 
The goal is to obtain snapshot images of bright objects, with the 351 simultaneous baselines, up to $600 \mathrm{~m}$ long, genrated by the 27 telescopes. We also see the OVLA as a precursor of larger instruments using $8 \mathrm{~m}$ mirrors for example, and space versions which may at some stage be implemented on the Moon or on high Earth-orbits. Because the OVLA structure is highly modular, the en gineering concept does not have to be frozen at an early stage. Instead, the instrument is conceived as a test bench, in order to accomodate evolving implementations of the telescopes, the recombiner, etc.... This allows taking risks in terms of the techniques utilized. The latest components or ideas will be utilized. With respect to the earlier description given in Labeyrie et al., 1991, little has changed. The construction of a prototype telescope is now reaching the stage of mirror polishing and building the active cell. Annealed $1.5 \mathrm{~m}$ blanks can be produced at the rate of one every 10 days.

\section{Telescope optics}

The paraboloidal primary at $f / 1.75$ is followed by a small gregorian paraboloidal secondary providing an afocal output towards the flat mirror located at the center of the spherical mount. This mirror is angularly driven to provide a stable coudé beam reaching the central station where the 27 beams are recombined.

Because of the narrow coudé field, amounting to arc-seconds, it is of interest to utilize the primary focus of the telescopes for various monitoring purposes, and possibly for a field-slicer arrangement allowing the simultaneous observation of a reference star together with the main object. It may be achieved with a CCD camera exploiting the primary field around a pinhole selecting the main object.

\section{Beam recombiner}

The central optics will be interchangeable, by means of tables resting on indexing supports. Among the designs currently considered is the folded-collector arrangement represented in figure 2 .

It is intended to allow simultaneous observation in the visible and the infra-red, while using a minimal number of mirrors. Field rotation is corrected by rotating part of the optical train.

The small lenses are infra-red transmitting. Additional components may be added for polarisation control, etc.... We plan to implement fast partial adaptive optics in this recombiner, as proposed by F. Roddier (1991). GI2T.

A prototype table is to be constructed along these lines for the 

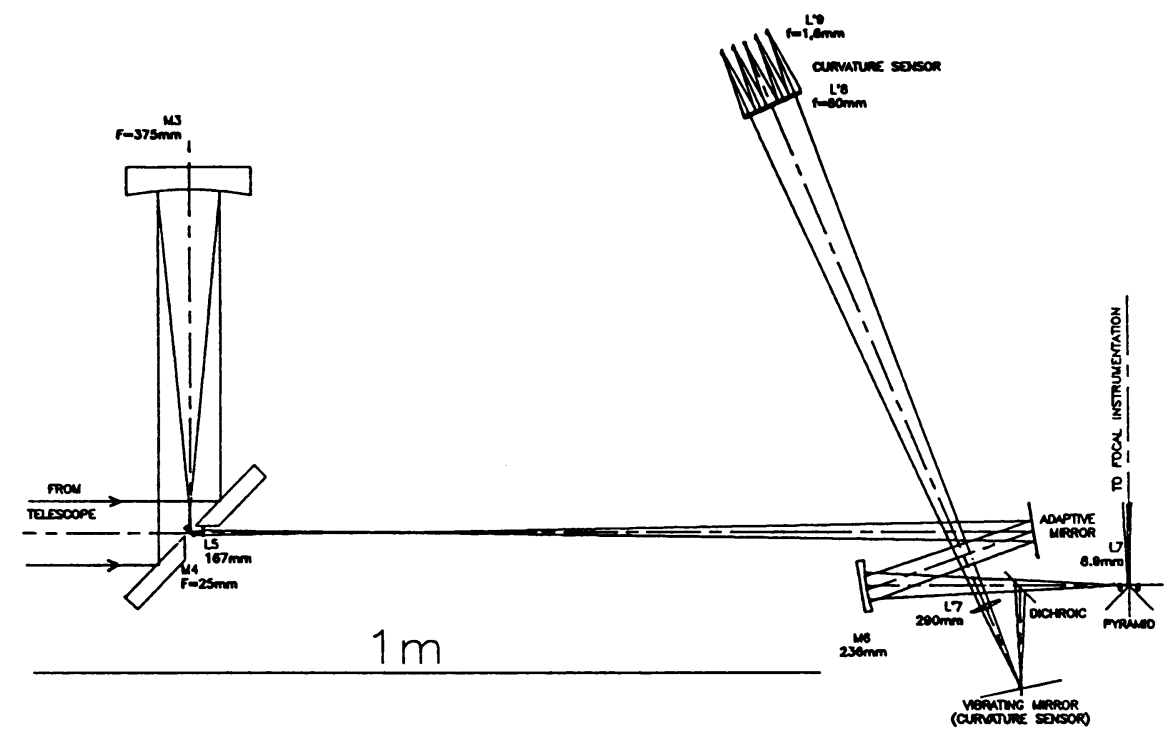

Figure 2: Beam recombiner (one beam). The parallel beam from the telescope is sent to an afocal beam reducer made of the $45^{\circ}$ flat mirror, the parabolic mirror M3 and the off-axis parabolic mirror M4. Field rotation is corrected by rotating this reducer around its input/output axis. The pupil is imaged on the adaptive deformable mirror by lens L5. It is then reduced and reimaged by L6 and L7 on the pyramid mirror which combines the 27 beams. Part of the light after the deformable mirror is sent to the wavefront analyser (curvature sensor).

\section{Mirror fabrication}

Low-cost industrial glass, available in $25 \mathrm{~mm}$ thickness, appears usable as a mirror material if an active cell is utilized. Indeed, the old problem of thermal deformations is likely to vanish if the mirror is a thin meniscus with active supports. The construction of a prototype active mirror cell is under way.

We have started producing meniscus blanks from $25 \mathrm{~mm}$ plate glass, by slumping disks in an oven. An oven was built by two of us (BD and JD), and fitted with a paraboloidal concave mold. The mold is made of refractory plaster containing alumina fiber and silica powder. Its paraboloidal shape was carved with a parabolic blade rotating on a vertical axis. The oven structure was designed to minimize the deformations of the mold durig the heating cycle. 
Flat $1.52 \mathrm{~m}$ disks, some of them with a central hole of $350 \mathrm{~mm}$, are cut from $25 \mathrm{~mm}$ plate glass by a commercial glass supplier. We slump them in the mold at $650^{\circ} \mathrm{C}$ and then anneal them during a week by lowering the temperature according to a programmed law. Measurements of residual stresses in polarized light, kindly made by J.Texereau, have shown a low level of residual stresses corresponding to an optical path difference of $19 \mathrm{~nm} / \mathrm{cm}$.

Three blanks have already been produced. One was accidentally dropped during handling and broken. The last blank approaches the best paraboloid within a few tenths of a millimeter.

We have also briefly considered techniques for actively controlled slumping, which may at some stage provide mirrors requiring no polishing. The idea is to control the shape of the viscous glass in the oven, possibly spinning, using active supports. and on-line optical testing It is unclear yet whether it may at some stage compete with traditional figuring techniques.

One blank has been delivered to the Observatoire de Marseille, for a polishing attempt utilizing the stressing methods developped by Lemaître, described in Labeyrie et al. (1988). The blank will be held by its edge, using strain gages to achieve a uniform load while the under side will be pushed by compressed air. The method appears promising for the fast figuring of numerous mirrors. However, it does not accomodate the central hole.

Steps are also taken to try on a second blank.a different figuring method, involving excentered polishing strokes to generate the paraboloidal shape.

One of us (AL) has also conceived a third parabolisation method, using a full-size segmented tool undergoing active deformations, as sketched in fig. 3. The principle is that a paraboloidal function added to a wedge-shaped function is an identical but translated paraboloidal function. A special, active, polishing tool such as shown in fig. 3 has been designed and partially built by one of us (JD). The mirror being held fixed, facing down, the polishing strokes applied to the tool cause it to reshape itself as required to match the paraboloidal mirror. This active deformable tool is very stiff, and its shape variation is determined by the combined translation and tilting of the tool segments.

The method is mathematically rigorous, and therefore capable of generating even very fast mirrors in principle, but it is unclear yet whether it may prove preferable to the other methods previously mentioned, given the moderate focal ratio considered here.

It was initially considered that the techniques of mirror replication, using thin layers of epoxy resin, would be most suitable for producing the numerous mirrors required for the OVLA. Good results have been obtained at Nice by P.Assus, A.Glentzlin and their collaborators for 
mirrors up to one meter in size. Replication may prove of interest for $1.5 \mathrm{~m}$ mirrors, and larger mirrors up to perhaps $8 \mathrm{~m}$ at some stage. However, the recent progress in the art of figuring strongly aspheric surfaces makes it worthwhile to experiment both approaches.

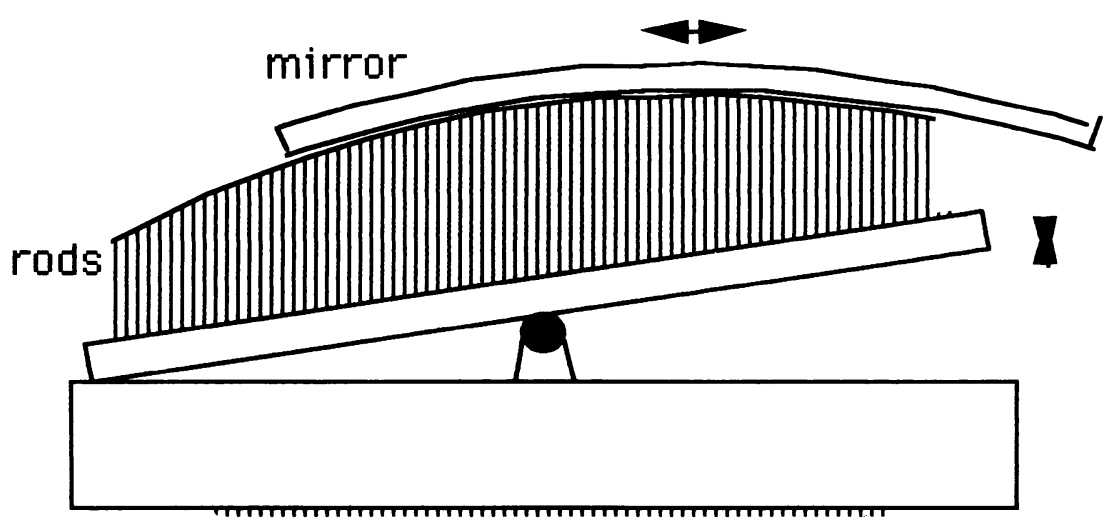

Figure 3: Active polishing tool for paraboloidal mirrors. The mirror is facing down. The pitch is carried by a series of rods maintained vertical, at constant spacing, while their flat supporting table is tilted in proportion to the amplitude of the polishing stroke applied to the mirror. The pure vertical translation of the rods causes their upper end to constantly match the paraboloidal surface of the mirror.

\section{Active mirror cell}

The $1.5 \mathrm{~m}$ mirrors have nearly the same aspect ratio as the $8 \mathrm{~m}$ mirrors of the VLT. They similarly require active cells to maintain their parabolic shape with the required accuracy. Because adaptive mirrors, in addition to the active primary, are also expected to become available for the OVLA, the optical tolerances on the primary mirror figure can be somewhat relaxed.

We have tried to produce a simplified design for an active supporting system: calculations of the flexural behaviour, by one of us (LA), using both analytical and finite-element methods, have shown that 32 supports, each having a triple contact point, suffice to achieve a wave figure quality reaching $32 \mathrm{~nm}$ peak to valley and $9 \mathrm{~nm}$ RMS. No astatic levers are used. Small commercial motors with a reducer and screw 
(Portescap) provide the required forces against a spring which carries the supporting triplet plate. The forces are measured with strain gages.

Designs for active lateral supporting are also considered.

\section{Mounts}

Following tests of the novel spherical mount built at Haute Provence by $\mathrm{CC}$, previously reported in Labeyrie et al. (1991), we have undertaken to complete the mount. The fiberglass sphere which carries the optics must have a slot on its side for extracting the coudé beam. This significantly weakens the sphere, and increases its flexure. To minimize the flexure, while also sealing the sphere against rain and wind, a system of bridge-shutters has been designed by one of us (CC). A test version of the system, under construction, is going to be installed on the prototype telescope.

\section{Metrology}

The laser system for the OVLA ( Morand et al. 1991) is under development and experimentation by one of us (FM) on one of the GI2T telescopes. The sensitivity for a first-generation system is expected to be of the order of 0.1 micron error for longitudinal distance measurements and 2 microns for transverse measurements on short-baselines .

\section{Adaptive optics, laser shooting and field-slicer}

Pending the development of concave adaptive mirrors, for use as a Gregorian secondary, we plan to use a flat adaptive corrector in the central recombining optics. The design introduced by Roddier (1991) is of particular interest, and we expect to implement a version optimized for the OVLA telescopes. Each telescope will have its dedicated corrector, providing a phased sub-image from the corresponding telescope, and there will be additional "piston" mirrors for phasing the telescopes.

The basic adaptive system just mentioned is suitable for bright stars, up to the 10th-13th stellar magnitude in the visible, but not for fainter sources. These may require additional, and rather complex, optics: 1a field slicer at the primary focus of each telescope, in order to produce two coudé beams, respectively from the main object and from a reference star up to an arc-minute apart; 2- a laser shooting system from each telescope towards the faint object.

It is however unclear yet wheter adaptive observing is practically feasible on faint objects. Provisions are made for implementing the 
faint-obect phasing at a later stage, if it proves feasible and if the scheduling of interferometer projects in space remains so slow as to justify observations of faint objects from the ground.

\section{Telescope translators (hexapod robots)}

Two of us are involved in the development of the hexapod translators which will slowly move the telescopes during the observation. A working model of one leg has been built by one of us (JPR), and JK is investigating hierarchical architectures of microcontrollers and software for driving the hexapods.

A subsequent step will be the construction of full-size elements for the legs. Once qualified, in terms of the operating performance, vibration levels, etc.. a complete hexapod translator will be built.

One of the issues is the implementation of an energy-efficient mechanism. Because slow motors applying high torques have a low energetic efficiency, until room-temperature supra-conductive coils become available, faster motors would seem preferable. However, the gears required for speed reduction cause vibrations. It may also be attempted to reduce the required torques by balancing the "insect" structure and obtain a self-standing static equilibrium. Passive pistons transferring efforts between legs can do it, but cables or gears may be preferable.

Another point of interest is the choice between walking on an array of posts, or on natural ground. The former requires simple forms of artificial vision, as demonstrated for example by Franceschini and hus group from their study of fly's eyes. The latter may require no vision if the ground is reasonably smooth, but requires some sensing of ground pressure and settling. A very detailed mathematical modelling of insect motion for robotics, made by Eltze, Weidemann \& Pfeiffer (1992), is partially applicable to the present situation.

\section{Post-OVLA ideas: ground and space}

Because the established optical shops which have undertaken the figuring of $8 \mathrm{~m}$ mirrors will somehow demand continuing orders, it seems likely that larger OVLA's, with $8 \mathrm{~m}$ subapertures, will be the next step.

If space sites become favored, instruments such as the proposed Lunar Optical Very Large Array may become built. A tethered system was recently proposed by Claudin and Labeyrie (1992) for orbital sites. 


\section{Conclusion}

The construction of the Optical Very Large Array is proceeding at a reasonable pace. The prototype telescope is expected to see first-light in a year, without its legs however. These will require perhaps another 6 months or a year. Once qualified, a series of telescopes will have to be built. It is likely that the required funding will be available at this stage. Participations from other groups are welcome.

\section{References}

Claudin Ph., Labeyrie A.:1992 in proc. ESA Colloquium on Targets for Spacebased Interferometry, Beaulieu, France, Octobre.

Eltze, J., Weidemann, H. \& Pfeiffer, F.: 1992 in proc. Int. Symposium Missions, Technologies and Design of Planetary Mobile Vehicles, Toulouse, sept. CNES/Cepadues.

Labeyrie A., Lemaître G., Thom C., Vakili F.: 1998 in proc. ESO Conf High Resolution Imaging by Interferometry, Garching.

Labeyrie A., Cazalé C., Gong S., Morand F., Mourard D., Kessis J. J., Rambaut J. P., Vakili F., Vernet D., Arnold L.: 1991, High-resolution imaging by interferometry, ESO conference, Garching.

Morand F., Gong,S., Mourard, D. \& Labeyrie,A.: 1991, High-resolution imaging by interferometry, ESO conference, Garching.

Roddier F., Northcott M., Graves J.E.: 1991, Publ. Astron. Soc. Pac. 103, p. 131. 